\section{Fasciculus Cuneatus}

John E. Mendoza

Department of Psychiatry and Neuroscience, Tulane Medical School and SE Louisiana Veterans Healthcare System, New Orleans, LA, USA

\section{Definition}

Fiber tract consisting of axons from the dorsal nerve roots that mediate fine tactile discrimination (stereognosis), proprioception, and vibration sense for the upper extremities and upper portion of the trunk. Along with the fasciculus gracilis which carries similar sensory information from the lower extremities and trunk, these pathways constitute the posterior (dorsal) columns of the spinal cord. Both are ipsilateral pathways (sensory information comes from the same side of the body as the tracts themselves). The fasciculus cuneatus synapses in the nucleus cuneatus in the medulla, where secondary fibers eventually cross the midline forming the medial lemniscus which terminates in the thalamus. Spinal cord lesions involving this pathway would result in ipsilateral upper extremity disturbances in position sense, vibration, two-point discrimination, or other stereognostic abilities such as being able to discriminate numbers traced on the skin or denominations of coins placed in the hand.

\section{Cross-References}

Dorsal Nerve Roots

Posterior Columns

\section{References and Readings}

Mendoza, J. E., \& Foundas, A. L. (2008). The somatosensory systems. In J. E. Mendoza \& A. L. Foundas (Eds.), Clinical neuroanatomy - A neurobehavioral approach (pp. 23-47). New York: Springer. 